\title{
Zukünftiges Honorar für Palliativmedizin sichern
}

\section{Hier steht eine Anzeige.}

Mit großer Mehrheit hat der Bundestag kürzlich einem neuen Hospiz- und Palliativgesetz zugestimmt. Im ambulanten Bereich soll der Gemeinsame Bundesausschuss (G-BA) demnach Anforderungen für die Palliativpflege festlegen. Ärzte, die sich an einem Fallmanagement an der Schnittstelle von palliativer und hospizlicher Versorgung beteiligen, sollen künftig neue Vergütungsleistungen erbringen und abrechnen dürfen, die von den Kassen extrabudgetär bezahlt werden müssen. Jährliche Mehrausgaben in „mittlerer zweistelliger“ Millionenhöhe sind hierfür eingeplant.

\section{MMW-KOMMENTAR}

Die neuen Leistungen werden vermutlich - im Gegensatz zu den bisher im EBM vorhandenen Abrechnungspositionen - an bestimmte Qualifikationsnachweise gebunden. Gedacht ist an ein Curriculum, wie es z. B. die Deutsche Gesellschaft für Palliativmedizin (DGP) oder die Bundesärztekammer bereits anbieten. Üblicherweise gibt es in solchen Fällen Übergangsbestimmungen, die sich daran orientieren, inwieweit sich die Praxis an der bisherigen Palliativversorgung beteiligt. Auch wenn Palliativleistungen aktuell in vielen KVen im Regelleistungsvolumen (RLV) oder im qualifikationsgebundenen Zusatzvolumen (QZV) budgetiert sind, lohnt es sich deshalb schon heute, diese Leistungen nicht nur zu erbringen, sondern auch differenziert abzurechnen. Bei der zu erwartenden Erteilung der Abrechnungsgenehmigung für die neuen Leistungen kann sich das durchaus auszahlen.

Tab. 1 Diese palliativmedizinischen Leistungen im EBM können sich schon bald ",auszahlen“ - wenn man sie heute schon differenziert abrechnet

\begin{tabular}{|l|l|l|}
\hline EBM & Legende & Euro \\
\hline 03370 & $\begin{array}{l}\text { Palliativmedizinische Ersterhebung des Patienten- } \\
\text { status inklusive Behandlungsplan, einmal im Krank- } \\
\text { heitsfall }\end{array}$ & 35,03 \\
\hline 03371 & $\begin{array}{l}\text { Zuschlag zur Versichertenpauschale Nr. 03000 für } \\
\text { die palliativmedizinische Betreuung des Patienten } \\
\text { in der Arztpraxis, einmal im Quartal }\end{array}$ & 16,33 \\
\hline 03372 & $\begin{array}{l}\text { Zuschlag zu den Nrn. 01410 oder 01413 für die } \\
\text { palliativmedizinische Betreuung in der Häuslichkeit, } \\
\text { je 15 Minuten }\end{array}$ & 12,74 \\
\hline 03373 & $\begin{array}{l}\text { Zuschlag zu den Nrn. 01411, 01412 oder 01415 } \\
\text { für die palliativmedizinische Betreuung in der } \\
\text { Häuslichkeit, je Besuch }\end{array}$ & 12,74 \\
\hline & & \\
\hline
\end{tabular}

